\title{
SINR-based $k$-coverage probability in cellular networks with arbitrary shadowing
}

\author{
H.P. Keeler ${ }^{\dagger}$, B. Błaszczyszyn ${ }^{\dagger}$ and M. K. Karray*
}

\begin{abstract}
We give numerically tractable, explicit integral expressions for the distribution of the signal-to-interference-andnoise-ratio (SINR) experienced by a typical user in the downlink channel from the $k$-th strongest base stations of a cellular network modelled by Poisson point process on the plane. Our signal propagation-loss model comprises of a power-law pathloss function with arbitrarily distributed shadowing, independent across all base stations, with and without Rayleigh fading. Our results are valid in the whole domain of SINR, in particular for SINR $<1$, where one observes multiple coverage. In this latter aspect our paper complements previous studies reported in [1].

Index Terms-Wireless cellular networks, Poisson process, shadowing, fading, SINR, multiple coverage, symmetric sums.
\end{abstract}

\section{INTRODUCTION}

Shannon's theory and its modern extensions quantify the quality of communications channels (ergodic capacity, finite block errors, error exponents, etc.) in a probabilistic manner by considering averages over codewords and channel characteristics (noise, fading, etc.). For communication networks with many channels, it has been recently suggested to use a stochastic geometric approach [2] consisting in taking spatial averages over node (emitter, receiver) locations. Establishing clear connections between stochastic-geometric averages and basic information-theoretic notions may be difficult (cf e.g. [3]) but this approach for wireless networks has recently attracted a lot of attention. In particular, the fundamental characteristic, discovered in information theory, signal-to-noise-ratio is now being studied in many geometric contexts with the incorporation of interference. This paper contributes to this approach by considering the distribution of the signal-to-interference-andnoise-ratio (SINR) of a typical user on down-link channels from different base stations of a single-tier cellular network modelled by Poisson point process on the plane. In particular, it complements [1], by providing explicit characterization of low (less than one) values of SINR. Current cellular-network technology allows for effective use of such SINR regimes, whence our motivation comes.

Cellular network models based on the Poisson point process have been shown to give tractable and accurate solutions [4], with the Poisson assumption being justified by representing highly irregular base station deployments in urban areas [5] or mimicking strong log-normal shadowing [6], or both. Knowledge of the distribution of SINR allows to calculate key performance indicators of cellular networks, e.g. spectral efficiency [7] ${ }^{1}$ or energy efficiency [6,9]. It can also be (non-

${ }^{\dagger}$ Inria/Ens, 23 av. d'Italie 75214 Paris, France

*Orange Labs, 38/40 rue Général Leclerc, 92794 Issy-Moulineaux, France

${ }^{1}$ From $[8, \S 4.2 .2]$ we know that it represents the critical traffic demand per base station, beyond which the best-effort service of variable-bit-rate traffic becomes unstable. trivially) related to user-level quality-of-service metrics.

In studying the SINR of a given user with respect to the base station with the greatest received signal, the main difficulty for small values of SINR is taking into account multiple coverage. Simple algebra shows that there can be at most one base station offering a given user SINR $\geq 1$, and hence the probability of having at least one station covering at this level reduces to the sum of probabilities of SINR-coverage over all base stations. However, this is not the case for $\operatorname{SINR}<1$, where one needs to study probabilities of simultaneous coverage by several base stations. We express these probabilities via the so-called symmetric sums and relate them to the appropriate partitioning of the SINR domain for SINR $<1$, which are the main ideas behind this paper.

Our signal propagation-loss model consists of the deterministic power-law path-loss function and independent (across all base stations), arbitrarily distributed shadowing. As previously observed $[6,10]$, any characteristic involving only the sequence of propagation-loss values experienced by a given user from all base stations entirely depend on the distribution of the shadowing via its moment of order $2 / \beta$, where $\beta$ is the pathloss exponent. We also study the impact of Rayleigh fading on the SINR coverage by incorporating into the propagation-loss model additional independent (across all base stations) random variables with exponential distributions. Assuming that fading affects the SINR coverage condition but not the choice of the serving base station, we observe that it worsens the coverage particularly at small values of SINR.

\section{Related work}

The SINR coverage in a multi-tier network was studied in [1] for SINR $\geq 1$. Two different approaches to express the distribution of the SINR in its whole domain, both involving inversion of Laplace transforms, were presented in $[6,11]$. Our expressions, which involve two key families of integrals (over the positive real line and a hyper-cube respectively) are much more tractable in numerical evaluation. Our model with fading was recently examined in [12] under slightly more general assumptions. We revisit it in order to present more closedform expressions under our specific assumptions, and compare the coverage probabilities obtained in it to these in the model without fading. ${ }^{2}$

\footnotetext{
${ }^{2}$ Which of these two models is more appropriate depends on the context. For example if users are motionless, then the fading should be taken into account, while highly mobile users "see" channel characteristics averaged over fading, cf [13, Proposition 3.1].
} 


\section{MODEL DESCRIPTION}

On $\mathbb{R}^{2}$, we model the base stations with a homogeneous Poisson point process $\Phi$ with density $\lambda$. Given $\Phi$, let $\left\{S_{x}\right\}_{x \in \Phi}$ be a collection of independent and identically distributed random variables, that represent the shadowing experienced between station $x \in \Phi$ and a typical user located, without loss of generality, at the origin. Let $S$ denote a generic shadowing variable. The distribution of $S$ is arbitrary except for a technical assumption $\mathbf{E}\left[S^{\frac{2}{\beta}}\right]<\infty$ and a conventional assumption that $\mathbf{E}[S]=1$ that we make without loss of generality.

\section{A. SINR multi-coverage}

We define the SINR of the typical user with respect to the station $x \in \Phi$ by

$$
\operatorname{SINR}(x):=\frac{S_{x} / \ell(|x|)}{W+I-S_{x} / \ell(|x|)},
$$

where the constant $W$ is the noise power, $I=\sum_{x \in \Phi} S_{x} / \ell(|x|)$ is the total power received from the entire network, and the path-loss function is

$$
\ell(|x|)=(K|x|)^{\beta},
$$

with constants $K>0$ and $\beta>2$. In this paper we are interested in the distribution of the coverage number of the typical user defined as the number of base stations that the typical user can connect to at the SINR level $T$, namely

$$
\mathcal{N}(T)=\sum_{x \in \Phi} \mathbf{1}[\operatorname{SINR}(x)>T]
$$

The probability of the typical user being covered by at least $k$ base stations, which we call $k$-coverage probability, is

$$
P_{c}^{(k)}(T)=\mathbf{P}\{\mathcal{N}(T) \geq k\} .
$$

In particular, the coverage probability of the typical user is $\left(^{3}\right)$ $P_{c}(T):=P_{c}^{(1)}(T)$.

Since the function $x /(A-x)=A /(A-x)-1$ is increasing in $x, P_{c}^{(k)}(T)$, as a function of $T$, is the tail-distribution function of the SINR experienced by the typical user with respect to the base station offering the $k$ th smallest propagation-loss $Y_{k}$ : $\left(Y_{k}\right)^{-1} /\left(W+I-\left(Y_{k}\right)^{-1}\right)$, where $Y_{1}<Y_{2}<\ldots$ is the process of order statistics of $\left\{\ell(|x|) / S_{x}: x \in \Phi\right\}$. In particular, $P_{c}(T)$ is the tail-distribution function of the SINR with respect to the base station with the smallest propagation-loss.

Related quantities of interest include also the expected converge number $\mathbf{E}[\mathcal{N}(T)]=\sum_{n=0}^{\infty} n \mathbf{P}\{\mathcal{N}(T)=n\}$ and its probability-generating function $G(z)=\mathbf{E}\left[z^{\mathcal{N}(T)}\right]$.

\section{B. Adding fading to the model}

In this extension of the previous model we assume that the propagation-loss of each base station $x \in \Phi$ is further modified by a random fading variable $F_{x}$ and equal to $l(|x|) /\left(S_{x} F_{x}\right)$, where given $\Phi,\left\{F_{x}\right\}_{x \in \Phi}$ is a collection of independent and identically distributed random variables, independent of shadowing $\left\{S_{x}\right\}_{x \in \Phi}$. In this paper we will assume Rayleigh fading, i.e., that the generic fading variable $F$ is exponential, with $\mathbf{E}\left[F_{x}\right]=1$. A key assumption is that fading perturbs the SINR

\footnotetext{
${ }^{3}$ This notation is similar to that of [1], which uses $\beta$ instead of $T$.
}

coverage condition but not the choice of the serving base station ${ }^{4}$. In consequence, the coverage probability under fading (with respect to the smallest fading-averaged-propagation-loss base station) is defined as

$$
\tilde{P}_{c}(T)=\mathbf{P}\left\{\frac{\left(Y_{1}\right)^{-1} F}{W+I-\left(Y_{1}\right)^{-1} F}>T\right\},
$$

where, recall, $Y_{1}$ is the smallest propagation-loss received by the typical user in the model without fading.

\section{PRELIMINARY OBSERVATIONS}

\section{A. Invariance with respect to the shadowing distribution}

Lemma 1: [Cf [6, 10]] The fading-averaged-propagationloss process $\left(Y_{n}: n \geq 1\right)$, considered as a point process on the positive half-line $\mathbb{R}^{+}$is a non-homogeneous Poisson point process with intensity measure $\Lambda([0, t))=a t^{\frac{2}{\beta}}$ where

$$
a:=\frac{\lambda \pi \mathbf{E}\left[S^{\frac{2}{\beta}}\right]}{K^{2}} .
$$

Consequently, the distribution of $\left(Y_{n}: n \geq 1\right)$, and hence the functions $P_{c}^{(k)}(T)$ and $\tilde{P}_{c}(T)$, depend on the model parameters (including the shadowing $S$ distribution) only though the noise level $W$, path-loss exponent $\beta$ and the constant $a .^{5}$

\section{B. Symmetric sum representation}

For any given $T$ and $n \geq 1$ define the $n$th symmetric sum $\mathcal{S}_{n}(T):=\mathbf{E}\left[\sum_{\substack{x_{1}, \ldots, x_{n} \in \Phi \\ \text { distinct }}} \mathbf{P}\left\{\operatorname{SINR}\left(x_{i}\right)>T, i=1, \ldots, n \mid \Phi\right\}\right]$

where $\mathbf{P}\{\ldots \mid \Phi\}$ denotes the conditional probability given $\Phi$ (with random shadowing marks). We set $\mathcal{S}_{0}(T) \equiv 1$, and note that $\mathcal{S}_{n}(T)$ is the expected number of ways that the typical user can connect to $n$ base stations when there are $\mathcal{N}(T)$ base stations each with a SINR greater than $T$. We have the following identities related to the famous inclusionexclusion principle (cf e.g [14, IV.5 and IV.3] for (8) and (9), respectively). ${ }^{6}$

Lemma 2: We have for $k \geq 1$

$$
\begin{aligned}
P_{c}^{(k)}(T) & =\sum_{n=k}^{\infty}(-1)^{n-k}\left(\begin{array}{l}
n-1 \\
k-1
\end{array}\right) \mathcal{S}_{n}(T), \\
\mathbf{P}\{\mathcal{N}(T)=k\} & =\sum_{n=k}^{\infty}(-1)^{n-k}\left(\begin{array}{l}
n \\
k
\end{array}\right) \mathcal{S}_{n}(T), \\
\mathbf{E}\left[z^{\mathcal{N}(T)}\right] & =\sum_{n=0}^{\infty}(z-1)^{n} \mathcal{S}_{n}(T), \quad z \in[0,1] \\
\mathbf{E}[\mathcal{N}(T)] & =\mathcal{S}_{1}(T) .
\end{aligned}
$$

\footnotetext{
${ }^{4}$ In other words, the user compares the received signals averaged over fading effects, which is justified by short time and space coherence properties of the (multipath) fading.

${ }^{5}$ This means that evaluating our quantities of interest in our model with a general distribution of shadowing $S$ and some value of the constant $K$, one can equivalently and for mathematical convenience assume some particular distribution $\tilde{S}$ of shadowing, e.g. exponential or constant, and $\tilde{K}=1$ provided one replaces $\lambda$ by $\lambda(\tilde{K} / K)^{2} \mathbf{E}\left[S^{\frac{2}{\beta}}\right] / \mathbf{E}\left[\tilde{S}^{\frac{2}{\beta}}\right]$ in the obtained formula. We will use these two representations of the general models when evaluating $\tilde{P}_{c}^{(k)}(T)$ and $P_{c}(T)$, respectively, in Sections IV-B and IV-C.

${ }^{6} \mathrm{~A}$ general relation between the distribution of $\mathcal{N}$ and the symmetric sums is given by the Schuette-Nesbitt formula, often used in insurance mathematics, cf [15].
} 
Our goal in Section IV-B will be to evaluate symmetric sums $\mathcal{S}_{n}(T)$, which will allow us to express easily our quantities of interest appearing in the right-hand side of the above expressions. Before doing this, in the following section we explain that the (apparently infinite) summations presented above boil down to finite sums, as for any given $T$ we have $\mathcal{S}_{n}(T)=0$ for $n$ large enough.

\section{Partition of the T-domain}

For real $x$ denote by $\lceil x\rceil$ the ceiling of $x$ (the smallest integer not less than $x$ ).

Lemma 3: For $n \geq 1, \mathcal{S}_{n}(T)=0$ whenever $T \geq 1 /(n-1)$. In other words, one can replace $\infty$ by $\lceil 1 / T\rceil$ in the sums in expressions given in Lemma 2.

Proof: This is stems from a well-known constraint of the SINR cell intersection. If the SINR of a given user with respect to $n$ distinct stations is to be larger than $T$, then $n T /(1+T) \leq$ 1 (cf [16, Proposition 6.2], with the strict inequality holding whenever there are other (interfering) stations or external noise, which is the case in our model. Hence, for $T \geq 1 /(n-1)$ all the terms (probabilities) in the $m$ th symmetric sum $\mathcal{S}_{m}(T)$ in (7) are null for every $m \geq n$.

\section{MAIN RESUlts}

\section{A. Key integrals}

We now introduce two families of functions which will allow us to express $\mathcal{S}_{n}(T)$ and, in consequence, the multi-coverage characteristics in the model without fading. For $x \geq 0$ define

$$
\mathcal{I}_{n, \beta}(x)=\frac{2^{n} \int_{0}^{\infty} u^{2 n-1} e^{-u^{2}-u^{\beta} x \Gamma(1-2 / \beta)^{-\beta / 2}} d u}{\beta^{n-1}\left(C^{\prime}(\beta)\right)^{n}(n-1) !}
$$

where

$$
C^{\prime}(\beta)=\frac{2 \pi}{\beta \sin (2 \pi / \beta)}=\Gamma(1-2 / \beta) \Gamma(1+2 / \beta) .
$$

Remark 4: We have

$$
\mathcal{I}_{n, \beta}(0)=\frac{2^{n-1}}{\beta^{n-1}\left(C^{\prime}(\beta)\right)^{n}} .
$$

The second family of functions are integrals over the hypercube. For $x \geq 0$ define

$$
\mathcal{J}_{n, \beta}(x)=\int_{[0,1]^{n-1}} \frac{\prod_{i=1}^{n-1} v_{i}^{i(2 / \beta+1)-1}\left(1-v_{i}\right)^{2 / \beta}}{\prod_{i=1}^{n-1}\left(x+\eta_{i}\right)} d v_{1} \ldots d v_{n-1}
$$

where $\eta_{i}:=\left(1-v_{i}\right) \prod_{k=i+1}^{n-1} v_{k}$.

Remark 5: For $\mathcal{J}_{2}$, a closed-form solution exists

$$
\begin{aligned}
& \mathcal{J}_{2, \beta}(x) \\
& =\frac{B(2 / \beta+1,2 / \beta+1)_{2} F_{1}(1,2 / \beta+1 ; 2(2 / \beta+1) ;-1 / x)}{x},
\end{aligned}
$$

where $B$ is the beta function [17, eq. 5.12.1] and ${ }_{2} F_{1}(a, b ; c ; z)$ is the hypergeometric function given by [17, eq. 15.11] (whose integral representation follows from eq. 15.1.2 and 15.6.1 therein). ${ }^{7}$

\section{B. Results for the model without fading}

For $0<T<1 /(n-1)$ define

$$
T_{n}=\frac{T}{1-(n-1) T} \text {. }
$$

We now present the key result for the model without fading, which gives an explicit expression for the symmetric sums $\mathcal{S}_{n}(T)$.

Theorem 6: Assume shadowing moment condition $\mathbf{E}\left(S^{2 / \beta}\right)<\infty$. Then

$$
\mathcal{S}_{n}(T)=T_{n}^{-2 n / \beta} \mathcal{I}_{n, \beta}\left(W a^{-\beta / 2}\right) \mathcal{J}_{n, \beta}\left(T_{n}\right)
$$

for $0<T<1 /(n-1)$ and $\mathcal{S}_{n}(T)=0$ otherwise, where $a$ is given by (6) and $T_{n}$ by (17).

Theorem 6 in conjunction of Lemma 2 and Lemma 3 give us in particular the following expression for the $k$-coverage probability:

Corollary 7: Under the assumptions of Theorem 6

$$
P_{c}^{(k)}(T)=\sum_{n=k}^{\lceil 1 / T\rceil}(-1)^{n-k}\left(\begin{array}{c}
n-1 \\
k-1
\end{array}\right) T_{n}^{-2 n / \beta} \mathcal{I}_{n, \beta}\left(W a^{-\beta / 2}\right) \mathcal{J}_{n, \beta}\left(T_{n}\right),
$$

The special case $k=1$, for $T \geq 1$ reduces this to expression [6, eq. (25)], which is in turn a special case of [1, eq. (2)] for a single-tier network.

Proof of Theorem 6: Assume $0<T<1 /(n-1)$ (otherwise the result follows from Lemma 3). Following the remark in Footnote 5 we will first evaluate $\mathcal{S}_{n}(T)$ assuming exponential distribution of $S$ and $K=1$, and then bring back the general assumptions appropriately rescaling the Poisson intensity $\lambda$. By the (higher order) Campbell's formula and the Slivnyak's theorem (see [16, (9.10) and (9.16)]) and a simple algebraic manipulation

$\mathcal{S}_{n}(T)=\frac{(2 \pi \lambda)^{n}}{n !} \int_{\left(\mathbb{R}^{+}\right)^{n}} \mathbf{P}\left\{\bigcap_{i=1}^{n}\left(\operatorname{SINR}^{\prime}\left(r_{i}\right)>T^{\prime}\right)\right\} r_{1} d r_{1} \ldots r_{n} d r_{n}$

where $\operatorname{SINR}^{\prime}\left(r_{i}\right):=\frac{S_{i} / \ell\left(r_{i}\right)}{\left(W+I+\sum_{j=1}^{n} S_{i} / \ell\left(r_{i}\right)\right)}$ and $T^{\prime}:=T /(1+T)$ with $I$ is as in (1) and $S_{i}$ exponential (mean-1) variables, mutually independent and independent of $I$. Moreover, the event whose probability is calculated in (19) is equivalent to

$$
\left\{\frac{\min \left(S_{1} / \ell\left(r_{1}\right), \ldots, S_{n} / \ell\left(r_{n}\right)\right)}{W+I+\sum_{j=1}^{n} S_{j} / \ell\left(r_{j}\right)}>T^{\prime}\right\} .
$$

For integer $i \in[1, n]$ denote $E_{i}:=S_{i} / \ell\left(r_{i}\right)$. By our previous assumption $E_{i}$ are independent exponential variables with means $1 / \mu_{i}=1 / \ell\left(r_{i}\right)$, respectively. Let $E_{M}:=$ $\min \left(E_{1}, E_{2}, \ldots, E_{n}\right)$. Note that $E_{M}$ is exponential variable with mean $1 / \mu_{M}=1 /\left(\sum_{i=1}^{n} \mu_{i}\right)$. Moreover, define the random variable $D:=\sum_{i=1}^{n} E_{i}-n E_{M}$. By the memory-less property

\footnotetext{
${ }^{7}$ We note that the form of $\mathcal{J}_{n, \beta}$ is similar to integral representations of the generalized hypergeometric function and a related integral generalization [18]. A closed-form solution of $\mathcal{J}_{n, \beta}(x)$ may exist, but that is left as a future task For low and intermediate $n$, regular numerical and Monte Carlo methods work well and give results in a matter of seconds on a standard PC machine; cf [19]. For high $n$, analysis of the kernel of $\mathcal{J}_{n}$ may lead to judiciously choosing suitable lattice rules, thus allowing for relatively fast integration [20].
} 
of the exponential distribution note the random variable $D$ is independent of $E_{M}$ and has a mixed exponential distribution characterized by its Laplace transform

$$
\mathcal{L}_{D}(\xi)=\frac{\prod_{i=1}^{n} \mu_{i}}{\sum_{i=1}^{n} \mu_{i}} \sum_{i=1}^{n} \frac{1}{\prod_{j=1, j \neq i}^{n}\left(\mu_{j}+\xi\right)} .
$$

Using the new random variables we can express the event (20) as $\left\{E_{M}>T_{n}(W+I+D)\right\}$ where $T_{n}$ is given by (17). Consequently, the probability $\mathbf{P}\{\ldots\}$ calculated in (19) is equal to $\mathcal{L}_{W}\left(\mu_{M} T_{n}\right) \mathcal{L}_{I}\left(\mu_{M} T_{n}\right) \mathcal{L}_{D}\left(\mu_{M} T_{n}\right)$, which is a product of three Laplace transforms. The first transform is simply $\mathcal{L}_{W}(\xi)=$ $e^{-W \xi}$, the second can be shown ([16, equation 2.25]) to be $\mathcal{L}_{I}(\xi)=e^{-\lambda \xi^{2 / \beta} \pi C^{\prime}(\beta) / K^{2}}$ while the last one is given in (21). After substituting the explicit path-gain function (2), noting that there is some symmetry in the integration variables $r_{i}$, changing the integration variables $s_{i}:=r_{i}\left(\lambda T_{n}^{2 / \beta} \pi C^{\prime}(\beta)\right)^{1 / 2}$ and replacing $\lambda$ by $a /(\pi \Gamma(1+2 / \beta))$ to revoke the exponential shadowing assumption and bring back the general distribution of shadowing and constant $K$ (cf Footnote 5) one obtains

$$
\begin{aligned}
& \mathcal{S}_{n}(T)=\frac{2^{n}}{T_{n}^{2 n / \beta}\left(C^{\prime}(\beta)\right)^{n}(n-1) !} \int_{0}^{\infty} \ldots \int_{0}^{\infty} \\
& \frac{e^{-\left(\sum_{i=1}^{n} s_{i}^{\beta}\right)^{2 / \beta}}\left(\prod_{i=1}^{n} s_{i}^{\beta+1} e^{-W(a \Gamma(1-2 / \beta))^{-\beta / 2} s_{i}^{\beta}}\right)}{\left(\sum_{i=1}^{n} s_{i}^{\beta}\right)\left(\prod_{i=2}^{n}\left[s_{i}^{\beta}+T_{n} \sum_{k=1}^{n} s_{k}^{\beta}\right]\right)} d s_{1} \ldots d s_{n} .
\end{aligned}
$$

A substitution of $n$-dimensional spherical-like variables (detailed in the appendix, Section A) completes the proof.

\section{Effects of Rayleigh fading}

We now consider the model with fading; cf Section II-B.

Theorem 8: The coverage probability under fading (defined in (5) is equal to

$$
\begin{aligned}
\tilde{P}_{c}(T) & =\frac{2}{\beta} \int_{0}^{\infty} t^{\frac{2}{\beta}-1} e^{-t T W a^{-\beta / 2}} e^{-t^{\frac{2}{\beta}}} \\
& \times \exp \left(-\frac{2}{\beta} \frac{T t^{2 / \beta}{ }_{2} F_{1}(1,1-2 / \beta ; 2-2 / \beta ;-T)}{(1-2 / \beta)}\right) d t,
\end{aligned}
$$

where, again, ${ }_{2} F_{1}(a, b ; c ; z)$ is the hypergeometric function mentioned in Remark 5 above.

Remark 9: The expression (24) can be easily evaluated numerically. Setting $W=0$ yields an analytic solution

$$
\tilde{P}_{c}(T)=\left[1+\frac{2}{\beta} \frac{{ }_{2} F_{1}(1,1-2 / \beta ; 2-2 / \beta ;-T)}{(1-2 / \beta)} T\right]^{-1} .
$$

Proof of Theorem 8: We use the propagation-loss process representation $\left\{Y_{n}\right\}$ defined in Section II-A (which does account for arbitrary general shadowing, but not for fading), which we enrich by independent exponential marking $F_{n}$ representing Rayleigh fading. By Lemma $1\left\{\left(Y_{n}, F_{n}\right): n \geq 1\right\}$ is independently marked Poisson point process of intensity $\Lambda(\cdot)$. Using this representation we can express the coverage probability of (5) as follows

$$
\begin{aligned}
\tilde{P}_{c}(T) & =\int_{0}^{\infty} \mathbf{P}\left\{F_{1} \geq s T\left(W+I_{(s, \infty)}\right)\right\} f_{Y_{1}}(s) d s \\
& =\int_{0}^{\infty} \mathcal{L}_{W}(s T) \mathcal{L}_{(s, \infty)}(s T) f_{Y_{1}}(s) d s,
\end{aligned}
$$

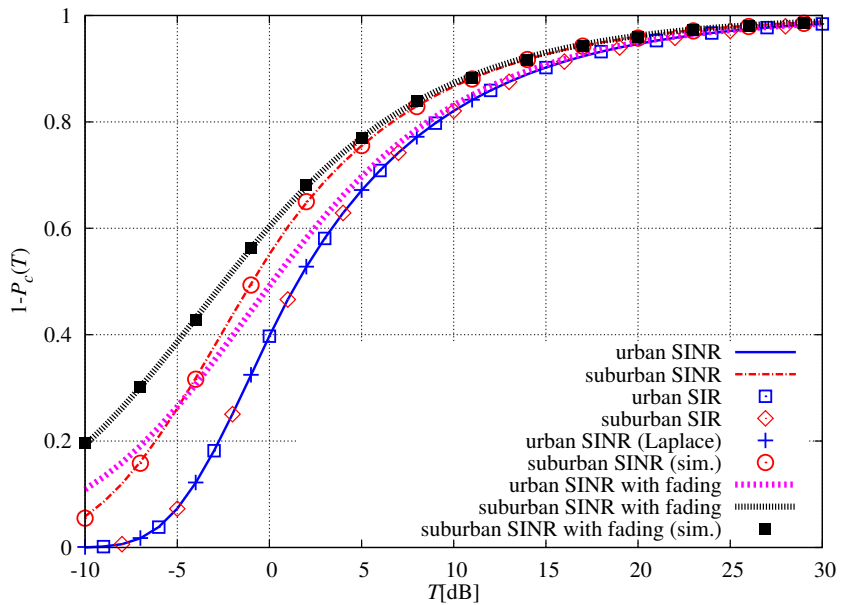

Fig. 1: Distribution function of SINR from the strongest base station with and without fading validated by the Laplace inversion method and simulation.

where $f_{Y_{1}}(s)$ is the probability density of $Y_{1}$, known to be (due to Poissonianity of $\left.\left\{Y_{n}\right\}\right) f_{Y_{1}}(d s)=-\frac{d}{d s} e^{-\Lambda(s)}=$ $2 a / \beta s^{2 / \beta-1}$ and where $I_{(s, \infty)}$ is the random variable representing conditional interference (accounting for shadowing and fading) given $Y_{1}=s$. Again, it is well known that $I_{(s, \infty)}$ is equal in distribution to $\sum_{Y_{n}>s} F_{n} / Y_{n}$ and has the Laplace transform which can be explicitly evaluated as follows

$$
\begin{aligned}
\mathcal{L}_{I_{(s, \infty)}}(\xi) & =\exp \left(-\int_{s}^{\infty}\left[1-\mathcal{L}_{F}(\xi / v)\right] \Lambda(d v)\right) \\
& =\exp \left(-\frac{2 a}{\beta} \frac{\xi s^{2 / \beta}}{s} \frac{{ }_{2} F_{1}(1,1-2 / \beta ; 2-2 / \beta ;-\xi / s}{(1-2 / \beta)}\right) .
\end{aligned}
$$

Plugging into (26) and substituting $t=s a^{\beta / 2}$ completes the proof.

\section{NUMERICAL ILLUSTRATIONS}

We use MATLAB implementation [19] for all our calculations. We set $\beta=3.8$ and $K=6910 \mathrm{~km}^{-1}$ (which corresponds to the COST Walfisch-Ikegami model for urban environment). The shadowing is modeled by a log-normal random variable of expectation 1 and logarithmic standard deviation $10 \mathrm{~dB}$ (cf [6]) which makes $\mathbf{E}\left(S^{2 / \beta}\right)=0.516$. We assume noise power $-96 \mathrm{dBm}$ normalized by the base station power $62.2 \mathrm{dBm}$ which makes $W=10^{-15.82}$. We consider two values for the density of base stations: $\lambda=4.619 \mathrm{~km}^{-2}$, which corresponds to a "urban" network deployment and $\lambda=0.144 \mathrm{~km}^{-2}$ for a "suburban" one. Figure 1 shows the distribution function of SINR from the strongest base station for both scenarios. We validate our approach by showing that the obtained results coincide with those of simulation and a Laplace inversion method developed in [6], with the latter approach being less numerically stable and much more time-consuming. We also plot the distribution of SIR in both scenarios (i.e. assuming $W=0$ ). Both SIR curves coincide with that of the SINR in urban area, thus showing that for urban density of stations the network is interference-limited, while for suburban density the impact of noise is non-negligible. Finally, we provide curves regarding the model with fading (i.e. $1-\tilde{P}_{c}(T)$ ). We observe that the impact of fading is non-negligible in both the urban 
and suburban scenario, and stochastically decreases SINR (the respective distribution functions are larger).

\section{CONCLUSION}

Cellular network models based on the Poisson point process allow for analytic expression for many important characteristics. Complementing previous studies, in this paper we give tractable, integral expressions (without any Laplace transform inversion) for the distribution of the SINR experienced by a typical user in the down-link channel from the $k$-th strongest base station in a single-tier cellular network. Our signal propagationloss model comprises of a power-law path-loss function with arbitrarily distributed shadowing, with and without Rayleigh fading.

\section{APPENDIX}

\section{A. Remaining proof of Theorem 6}

We introduce a change of variables inspired by the $n$ dimensional spherical coordinates (for example, see [21, eq. (1.3)])

$$
\begin{aligned}
s_{1} & :=u\left[\sin \theta_{1} \sin \theta_{2} \ldots \sin \theta_{n-1}\right]^{2 / \beta} \\
s_{2} & :=u\left[\cos \theta_{1} \sin \theta_{2} \ldots \sin \theta_{n-1}\right]^{2 / \beta} \\
s_{3} & :=u\left[\cos \theta_{2} \sin \theta_{3} \ldots \sin \theta_{n-1}\right]^{2 / \beta} \\
& \cdots \\
s_{n} & :=u\left[\cos \theta_{n-1}\right]^{2 / \beta} .
\end{aligned}
$$

Observe that $\sum_{i=1}^{n} s_{i}^{\beta}=u^{\beta}$ and $\prod_{i=1}^{n} s_{i}=u^{n}\left[\prod_{i=1}^{n} q_{i}\right]^{2 / \beta}$, where $q_{i}=q_{i}\left(\theta_{i}, \ldots, \theta_{n-1}\right):=\left(s_{i} / u\right)^{\beta / 2}$. When $\beta=2$ our system of coordinates boils down to the regular $n$-dimensional spherical coordinates, whose Jacobian is $\bar{J}\left(u, \theta_{1}, \ldots, \theta_{n-1}\right)=$ $u^{n-1} \prod_{i=1}^{n-1} \sin ^{i-1} \theta_{i}$; cf [21, eq. (1.5)]). By induction (or determinant properties and the chain rule) our coordinate system has the corresponding Jacobian

$$
\begin{aligned}
& J\left(u, \theta_{1}, \ldots, \theta_{n-1}\right) \\
& =\left(\frac{2}{\beta}\right)^{n-1} \bar{J}\left(u, \theta_{1}, \ldots, \theta_{n}\right)\left[\prod_{i=1}^{n-1} \sin ^{i} \theta_{i} \cos \theta_{i}\right]^{2 / \beta-1},
\end{aligned}
$$

which is clearly postive over the integration domain of interest. Denote $z:=W(a \Gamma(1-2 / \beta))^{-\beta / 2}$. The integral in (23) becomes

$$
\begin{aligned}
& \int_{0}^{\infty} \int_{[0, \pi / 2]^{n-1}} \frac{u^{n(\beta+1)}\left[\prod_{i=1}^{n-1} \sin ^{i} \theta_{i} \cos \theta_{i}\right]^{2(\beta+1) / \beta} e^{-u^{2}} e^{-z u^{\beta}}}{u^{n \beta} \prod_{i=2}^{n}\left[q_{i}^{2}+T_{n}\right]} \\
& \times J\left(u, \theta_{1}, \ldots, \theta_{n}\right) d u d \theta_{1} \ldots d \theta_{n-1} \\
& =\left(\frac{2}{\beta}\right)^{n-1} \int_{0}^{\infty} u^{2 n-1} e^{-u^{2}} e^{-z u^{\beta}} d u \\
& \quad \int_{[0, \pi / 2]^{n-1}} \frac{\prod_{i=1}^{n-1}\left[\sin ^{i} \theta_{i} \cos \theta_{i}\right]^{4 / \beta+1}\left[\sin \theta_{i}\right]^{i-1}}{\prod_{i \neq j}\left[q_{i}^{2}+T_{n}\right]} d \theta_{1} \ldots d \theta_{n-1} .
\end{aligned}
$$

The substitution $v_{i}=\sin ^{2} \theta_{i}$ makes the second integral (over the hypercube) equal to $2^{1-n} \mathcal{J}_{n, \beta}\left(T_{n}\right)$, which, after defining $\eta_{i}$, completes the proof in view of (23) and (17).

\section{REFERENCES}

[1] H. Dhillon, R. Ganti, F. Baccelli, and J. Andrews, "Modeling and analysis of K-tier downlink heterogeneous cellular networks," IEEE J. Sel. Areas Commun., vol. 30, no. 3, pp. 550-560, april 2012.

[2] M. Haenggi, J. Andrews, F. Baccelli, O. Dousse, and M. Franceschetti, "Stochastic geometry and random graphs for the analysis and design of wireless networks," IEEE J. Sel. Areas Commun., vol. 27, no. 7, pp. 1029-1046, September 2009.

[3] C. Galarza, P. Piantanida, and M. Kountouris, "On the block error probability of finite-length codes in decentralized wireless networks," in Proc. of Allerton Conference, sept. 2011, pp. 11951201.

[4] J. Andrews, F. Baccelli, and R. Ganti, "A tractable approach to coverage and rate in cellular networks," IEEE Trans. Commun., vol. 59, no. 11, pp. $3122-3134$, november 2011.

[5] C.-H. Lee, C.-Y. Shih, and Y.-S. Chen, "Stochastic geometry based models for modeling cellular networks in urban areas," Wireless Networks, pp. 1-10, 2012.

[6] B. Błaszczyszyn, M. Karray, and H. Keeler, "Using Poisson processes to model lattice cellular networks," in Proc. of IEEE INFOCOM, 2013, available also at http://arxiv.org/abs/1207.7208.

[7] M.-S. Alouini and A. Goldsmith, "Area spectral efficiency of cellular mobile radio systems," IEEE Trans. Veh. Technol., vol. 48, no. 4, pp. 1047-1066, jul 1999.

[8] T. Bonald, S. Borst, N. Hegde, and M. Jonckheere, "Flowlevel performance and capacity of wireless networks with user mobility," Queueing Systems, vol. 63, pp. 131-164, 2009.

[9] F. Richter, A. Fehske, and G. Fettweis, "Energy efficiency aspects of base station deployment strategies for cellular networks," in Proc. of IEEE VTC, sept. 2009.

[10] B. Błaszczyszyn and M. K. Karray, "Quality of service in wireless cellular networks subject to log-normal shadowing," IEEE Trans. Commun., vol. 61, no. 2, pp. 781-791, 2013, published on Early Access on December 2012.

[11] H. Dhillon, R. Ganti, F. Baccelli, and J. Andrews, "Coverage and ergodic rate in K-tier downlink heterogeneous cellular networks," in Proc. of Allerton Conference, sept. 2011, pp. 1627 -1632.

[12] T. T. Vu, L. Decreusefond, and P. Martins, "An analytical model for evaluating outage and handover probability of cellular wireless networks," in Proc of WPMC, sept. 2012, pp. $643-647$.

[13] S. Borst, "User-level performance of channel-aware scheduling algorithms in wireless data networks," in Proc. of IEEE INFOCOM, 2003, pp. 321-331.

[14] W. Feller, An Introduction to Probability Theory and its Applications, vol I., 3rd ed. New York: J. Wiley \& Sons, 1968.

[15] H. U. Gerber, Life Insurance Mathematics. Springer, 1995

[16] F. Baccelli and B. Błaszczyszyn, Stochastic Geometry and Wireless Networks, Volume I - Theory, ser. Foundations and Trends in Networking. NoW Publishers, 2009, vol. 3, No 3-4.

[17] (2012, Accessed on the 10th of September) Digital Library of Mathematical Functions. National Institute of Standards and Technology. Release 1.0.5 of 2012-10-01. [Online]. Available: http://dlmf.nist.gov/

[18] E. Ulanskii, "An identity for generalizing the hypergeometric integral," Mathematical Notes, vol. 79, no. 5, pp. 741-744, 2006.

[19] H. P. Keeler, "SINR-based $k$-coverage probability in cellular networks," MATLAB Central File Exchange, 2013. [Online] Available: http://www.mathworks.fr/matlabcentral/fileexchange/ 40087-sinr-based-k-coverage-probability-in-cellular-networks

[20] F. Kuo and I. Sloan, "Lifting the curse of dimensionality," Notices of the AMS, vol. 52, no. 11, pp. 1320-1328, 2005.

[21] D. Mustard, "Numerical integration over the n-dimensional spherical shell," Mathematics of Computation, vol. 18, no. 88, pp. pp. 578-589, 1964. 